\title{
Comparison of Three Polypyrrole-Cellulose Nanocomposites Synthesis
}

\author{
Benoit Bideau, Eric Loranger*, Claude Daneault \\ Lignocellulosic Materials Research Center, Université du Québec à Trois-Rivières, , Trois-Rivières, QC, Canada \\ Email: Eric.Loranger10uqtr.ca
}

\begin{abstract}
In this study, composite films based on TEMPO-oxidized cellulose nanofibers (TOCN), and polypyrrole (PPy) were synthesized by three different processes. The flexible composite films were investigated with scanning electron microscopy, thermogravimetric analysis, contact angle measurements, and finally, by mechanical and electrical testing. The developed composites have shown interesting mechanical properties (TOCN/PPy-3) as Young modulus (6.35 GPa) and tensile stress $(65.6 \mathrm{MPa})$ or electrical conductivity (TOCN/PPy-1; $51.6 \mathrm{~S} / \mathrm{cm}$ ) for further applications such as flexible electrode. From proposed methods, the grafting of $\mathrm{N}$-(3-aminopropyl)pyrrole was also interesting because it presented intermediate properties from all composites, and could represent a good compromise between the mechanical and electrical properties. Depending of their final application and by choosing an appropriate fabrication method, these composites could be considered in the design of high-performance electrodes for supercapacitor, battery, sensor or various packaging.
\end{abstract}

Keywords: Polypyrrole, cellulose nanofibers, mechanical and electrical properties, composite.

\section{Introduction}

Considering the growing interest on the development of nanoscaled materials and especially bionanocomposites, researches have been driven toward conducting polymers because of their functional properties, allowing them to be used in many application fields such as batteries, sensors, antistatic coating, etc.[1,2]. Among conducting polymers, a particular interest has been focused on PPy because of his high electrical conductivity and his great thermal and environmental stability[3]. Moreover, ease of synthesis through radical polymerization coupled with an inexpensive oxidizing agent such as ferric chloride, further increase interest in this polymer[4,5]. However, PPy alone presents poor mechanical properties which limit his applications[1]. This is why, several studies have been conducted to improve PPy physical properties through the synthesis of composite with PPy. Thus, considerable attention has been focused on a reinforcement of the PPy matrix with natural compounds such as bacterial and algal cellulose[7], dextrin[8], carboxymethylcellulose[9], chitosan[10] or wood cellulose[11,12,13]. These materials exhibit great interest as they all come from renewable resources.

Among them, cellulose presents a great interest, because it is the most abundant organic polymer on Earth. It is inexpensive, relatively easy to process, renewable, and biodegradable. Thus several studies have been conducted on the cellulose/PPy composites which are used in flexible energy devices, such as batteries, in ion exchange membrane or in gas and humidity sensors[14,15,16]. The majority of these studies use cellulose derived from Cladophora sp. algae or bacteria, and PPy were obtained by in situ chemical oxidative polymerization of pyrrole[17]. Moreover, the abundant hydroxyl groups on the cellulose fibers are favourable to immobilise PPy nanoparticles by hydrogen-bond or electrostatic interactions[18]. These composites are highly porous and can be used as electrode materials in environmentally friendly charge storage devices[17]. The highly specific area of cellulose material contributes to obtaining high loading content of PPy nanoparticles. However, coating substances with PPy particles remains a challenge, because the choice of the substrate[19] and the synthesis conditions[20] directly affect the composite properties and morphology.

In this work, we have chosen to use a TEMPO Oxidized Cellulose Nanofibers (TOCN) as reinforcement agent in our composite. The cellulose nanofibers obtained by TEMPO oxidation reactions are produced from the modification of the primary carboxylic groups of the cellulose[21,22]. Among 
advantages of using oxidized cellulose fibres are their large specific surface area, their microporous structure and their surface charges, for good adherence, and their high mechanical properties[23,24].

As previously mentioned, generally, the bonds achieved between PPy and the reinforcement agent, resulting from in situ chemical polymerisation, are weak electrostatic interactions or hydrogen bonds. The main goal of the current study was to compare three composites resulting from three different synthesis process. The first process was in situ chemical polymerization which results in the composite commonly found in the literature while the second process consisted in the grafting of 1-(2Cyanoethyl)pyrrole on TOCN before PPy polymerization. The 1-(2-Cyanoethyl)pyrrole offers the possibility of creating covalent bonds (amide) between carboxylic groups and the PPy which are strong bond[25]. Finally, the third method was carried out to synthesize multilayer composites films, which were obtained via polymerization of pyrrole on the surface of the TOCN film. The study will discuss the composites properties and also highlight potential application for each synthesis process.

\section{Materials and Methods}

\subsection{Materials}

Pyrrole $\left(\mathrm{C}_{4} \mathrm{H}_{5} \mathrm{~N}\right)$, 1-(2-Cyanoethyl)pyrrole $\left(\mathrm{C}_{7} \mathrm{H}_{8} \mathrm{~N}_{2}\right)$, iron (III) chloride $\left(\mathrm{FeCl}_{3}\right)$ and all other chemicals were purchased from Sigma Aldrich and used as received. Cellulose nanofibers gel (2.5\%) was obtained by TEMPO oxidation and sonication treatments of a commercial bleached Kraft wood pulp[21]. This oxidation chemically modifies the primary alcohol into a carboxyl functional group. The carboxyl rate was measured at $1440 \pm 40 \mathrm{mmol} / \mathrm{kg}$. The gel used is composed of micro and nanofibers. The ratio was estimated to $30 \%$ of micro and $70 \%$ of nanofibers with an average width and length of about $3.5 \pm 1.0$ and $306 \pm 112 \mathrm{~nm}$ respectively[22].

\section{$2.2 \quad$ Methods}

\subsubsection{Preparation of Composite}

Three protocols were experimented in order to fabricate TOCN/PPy composites. The description of the protocols is given in the following paragraphs while Figure 1 presents a schematic of all the methods. The same ratio of TOCN, pyrrole and iron chloride was used for each method.

The first method was in situ polymerization of pyrrole in a TOCN solution. The TOCN solution $(60 \mathrm{ml})$ at $0.5 \%$ and Pyrrole solution $(2 \mathrm{ml})$ at $0.028 \mathrm{~mol}$ were introduced in a beaker. Afterwards, the mixture was stirred for 10 minutes and the oxidant solution $\left(\mathrm{FeCl}_{3}\right)$ at $0.2 \mathrm{~mol} / \mathrm{l}$ was added. The mixture was kept under stirring for an additional 30 minutes before turning black, thus showing polymerization. Then the solution was filtered on a Büchner filter $(90 \mathrm{~mm}$ of diameter) and rinsed thoroughly with distilled water. The resulting film is then placed into a hydrochloric acid ( $\mathrm{HCl})$ solution of at $0.1 \mathrm{~mol} / \mathrm{l}$, according to the literature[26] to enhance the conductivity. Finally, the composite (TOCN/PPy-1) was dried between two hot plates at $80{ }^{\circ} \mathrm{C}$ and stored before characterisation.

The second method takes place in two successive steps: the grafting of 1-(2-cyanoethyl)pyrrole onto the TOCN and the polymerization of pyrrole on the surface of the fibers. The first step consists in the reduction of 1-(2-cyanoethyl)pyrrole to $\mathrm{N}$-(3-aminopropyl)pyrrole. The product was formed in agreement with the data gathered in the literature[27]. A yellow oily product was obtained and stored at $4{ }^{\circ} \mathrm{C}$ for further uses. The reduction was confirmed by $1 \mathrm{H}$ NMR (data not shown in this article) with a yield of about $90 \%[28]$. Next, N-(3-aminopropyl)pyrrole was introduced in a $\mathrm{pH} 7$ buffer solution containing CNF at a concentration of $0,5 \%$ and the whole solution was then mixed for 10 minutes. NHydroxysuccinimide (NHS) and 1-ethyl-[3-(dimethylamino)propyl]-3-ethylcarbodiimide (EDC) were added[28] to initiate the grafting. The quantities of N-(3-aminopropyl)pyrrole, NHS and EDC have been calculated in order to graft $25 \%$ of the total available TOCN carboxylic groups $(1440 \mathrm{mmol} / \mathrm{Kg})$. The PyTOCN solution was stirred for 3 hours. In a second step, PyTOCN suspension (60ml) at $0.5 \%$ concentration was mixed with $2 \mathrm{ml}$ of Pyrrole $(0.028 \mathrm{~mol})$ for 10 minutes. Iron Chloride solution at 0.2 $\mathrm{mol} / \mathrm{l}$ was added to initiate the polymerization. The mixture was stirred for 30 minutes before being filtered on a Büchner filter (90mm of diameter). The composite was thoroughly rinsed with distilled 
water and placed into a solution of $0.1 \mathrm{~mol} / \mathrm{l} \mathrm{HCl}$. Finally, the composite (TOCN/PPy-2) was dried between two hot plates at $80{ }^{\circ} \mathrm{C}$ and stored before characterisation.

The third method was based on multiple layer principle. A $0.5 \%$ solution of TOCN (60 ml) was poured in a $90 \mathrm{~mm}$ aluminium dish and dried for 72 hours at ambient air, to fabricate the TOCN film. Next, the film was immersed in a solution of $\mathrm{FeCl}_{3}$ in $0.2 \mathrm{~mol} / \mathrm{l}$ for $20 \mathrm{~min}$. Then, $2 \mathrm{ml}$ of pyrrole $(0.028$ mol) was added and distributed over the entire film. After $30 \mathrm{~min}$ of polymerization, the film has completely darkened and is then coated with PPy particles on the surface. The composite then was thoroughly rinsed with distilled water and placed into a $\mathrm{HCl}$ solution at $0.1 \mathrm{~mol} / \mathrm{l}$ for 72 hours. Finally, the multilayer composite (TOCN/PPy-3) film was dried between two plates at $80{ }^{\circ} \mathrm{C}$ and stored before characterisation.

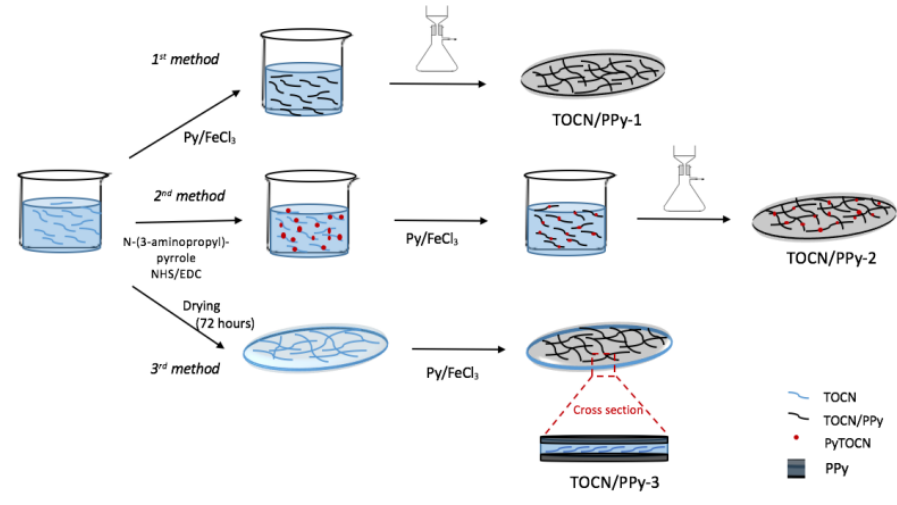

Figure 1: Schematic diagram of the three methods used to TOCN/PPy composite synthesis

\subsubsection{Characterization}

The composites were all conditioned in a controlled environment room $\left(25{ }^{\circ} \mathrm{C}, 50 \% \mathrm{RH}\right)$ during $24 \mathrm{~h}$ before all characterization. The thickness was measured by cross-sections image analysis from SEM micrograph. The ImageJ® software was used to obtain thickness average values of at least 5 measurements. Infrared spectra were recorded for each composite in ATR mode, at room temperature on a Nicolet IS10 FT-IR spectrometer (ThermoScientific) equipped with a Diamond/ZnSe crystal. Each spectrum was acquired in the range of $600-4000 \mathrm{~cm}^{-1}$. Duplicates of each sample were analyzed at five different points. The surface morphology of samples was studied using scanning electron microscopy (SEM) (JEOL-JSM 5500). Our composites did not need to be gold plated since they were sufficiently conductive. The roughness was analyzed with an Optical Profiler from Veeco Wyko (NT1100) with the 5.0X objective and 1.0 field of view (FOV). The measurement was done in VSI (Vertical scanning interferometry) mode. The average value of three replicates for each sample was collected. The tensile strength of the composites was measured on a universal testing machine (Instron 4201) at room temperature and controlled humidity. Elongation at break and Young's modulus of these samples were, therefore, determined from the stress-strain curves. Samples used in these measurements were cut from the casted film at $23 \mathrm{~mm}$ in length, $10 \mathrm{~mm}$ wide and a range of 0.04 to $0.10 \mathrm{~mm}$ in thickness. The average value of three replicates for each sample was collected. Thermal stability analysis of the samples was carried out in a Perkin-Elmer (Pyris Diamond) Thermoanalyzer. Each composite was heated in open alumina pans from 25 to $600{ }^{\circ} \mathrm{C}$, under a nitrogen atmosphere, at a heating rate of $10{ }^{\circ} \mathrm{C} / \mathrm{min}$. Then, samples were heated from 600 to $950{ }^{\circ} \mathrm{C}$ under air at a heating rate of $15{ }^{\circ} \mathrm{C} / \mathrm{min}$. The contact angles were determined using a Contact angle system OCA20 (DataPhysics). At least 5 drops (3E-2 $\mu \mathrm{l})$ of distilled water were deposited onto each substrate and a total 300 images were captured within 30 seconds for each drop. The electrical conductivity was measured at room temperature by a four-point probe method with the ResTest apparatus from Jandel Engineering Ltd. The probe was equipped with four tungsten carbide needles having a diameter of $100 \mu \mathrm{m}$ and spaced $1 \mathrm{~mm}$ apart. Resistance, R (ohms); length, L (cm); width, $\mathrm{w}(\mathrm{cm})$ and thickness, $\mathrm{t}(\mathrm{cm})$ were used to calculate the specific resistivity $(\rho)$ and the conductivity (r) in $\mathrm{S} \mathrm{cm}^{-1}$ using respectively the formula (1) and (2). The average value of three replicates for each sample was collected and reported as results. 


$$
\begin{aligned}
& \rho=R \cdot \frac{L}{w \cdot t} \\
& r=1 / \rho
\end{aligned}
$$

\section{Results and Discussion}

Composites characteristics and properties are directly influenced by their production method. Thus, we wish to highlight the impact that can have three different methods on TOCN/PPy composites. All the methods used, required the presence of an oxidant (iron chloride), to initiate the polymerization of pyrrole to PPy. The polymerization resulted in a color change as the solution is turning black a few seconds after the addition of the oxidant. The first and second method used consisted in the polymerization of the pyrrole around cellulose fibers before the solution filtration on Büchner funnel (Figure 1). The first one is being carried out by electrostatic interactions, while the second one uses a covalent bond between the polypyrrole particles and the cellulose fibers through the N-(3aminopropyl)pyrrole. The third method is different because, first, we synthesize a cellulose film before polymerising the pyrrole on the film surface.

\subsection{Morphological Structure}

Scanning electron microscopy (SEM) images of each composite film were analysed and shown in Figure 2. For starters, we can see that all samples were completely covered by polypyrrole particles. PPy morphology could be described as "muddy" for TOCN/PPy-1 and TOCN/PPy-2 (Figure 2a and 2b). The surface of the polypyrrole particles exhibits compact and homogeneous mud-like morphology. On Figure 2a, it is possible to see that cellulose fibers have completely disappeared by being covered with the polypyrrole particles. Large particles, with diameters of 100-200 nm, are visible and seem to be uniformly aggregate on the fibers. If we compare to Figure 2b, the morphology is a little different because we can see cellulose fibers shadow under the polypyrrole layer. However, all visible fibers are covered with polypyrrole particles. We suppose that PPy particles are closer to the cellulose fibers, with the presence of covalent bonds, which create a more wrinkled morphology. For TOCN/PPy-3, the morphology is relatively different and seems to be more planar. Indeed, it can be deduced that a TOCN film is covered by a thin layer of PPy particles. Moreover, the structure of a TOCN film is relatively close and compact, so all of the pyrrole solution will not be able to diffuse deep in the surface layer. This is why, the PPy chains were mostly on the film surface as demonstrated by Bideau \& al. [29]. This method creates a dense cellulose network which offers higher flexibility and mechanical properties to the composite, but a polymerization made on the surface, does not lead to a uniform layer on the fibers surface (Figure 2c). The SEM images attest that some pores are visible despite PPy particles. This one could be negatively affected the electrical properties. Finally, the morphology of these three composites seem relatively different due to the process used. This will have a direct impact on electrical and mechanical properties. The SEM images suggest a better PPy coverage for the TOCN/PPy-1 and TOCN/PPy-2 than TOCN/PPy-3.

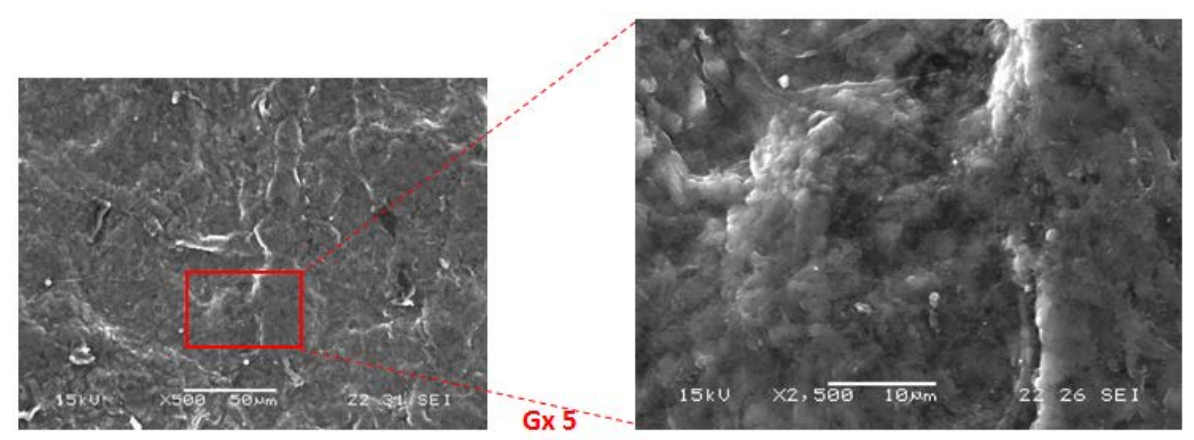

a) First method 


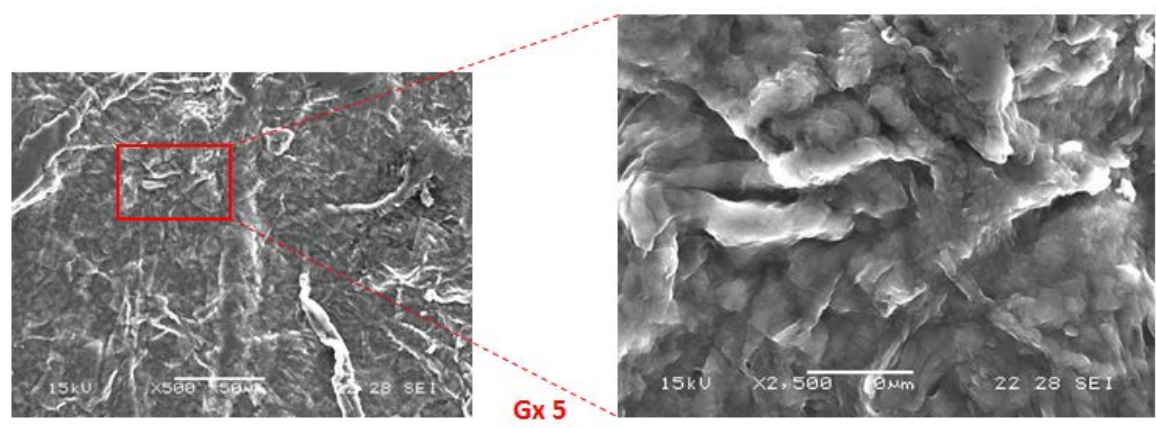

b) Second method

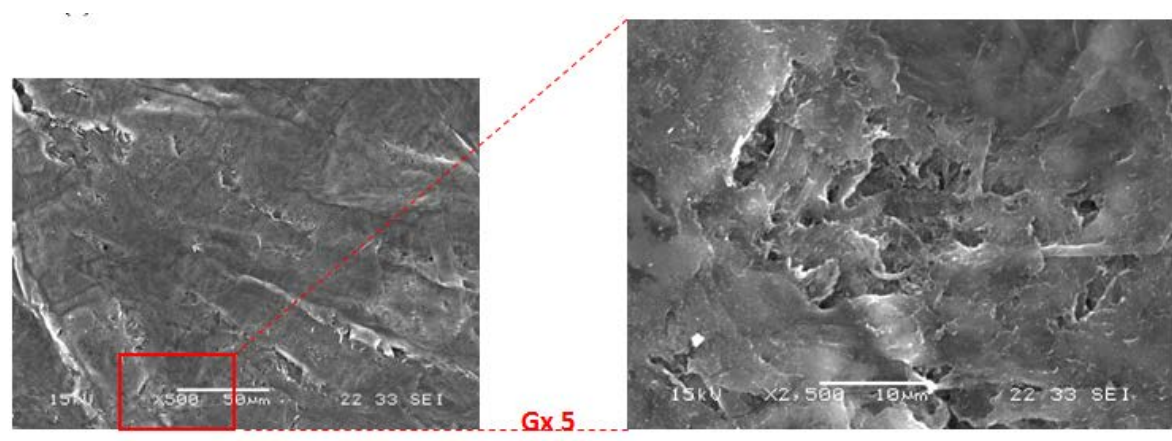

c) Third method

Figure 2. SEM images of TOCN/PPy composites made with three different methods: (a) TOCN/PPy-1 made with the first method; (b) TOCN/PPy-2 made with the second method; (c) TOCN/PPy-3 made with the third method

As expected, composites show variable roughness (Table 1). TOCN/PPy-1 and TOCN/PPy-2 present the most important roughness of three composites. In situ polymerization followed by Buchner filtration has led to wrinkled arrangement as observed on the previous SEM images. Filtration does not achieve an ordered arrangement of fibers, creating a steeped topography. Some PPy particles aggregate will increase the roughness despite that PPy particles may level the surface, by filling the pores. For this reason, these two composites have a much greater roughness than TOCN/PPy-3. Indeed, in the third method the TOCN films were synthesised before polymerization. The fibers have sedimented during $72 \mathrm{~h}$ (drying) which has led to planar surface. The polymerization creates a uniform PPy layer on the surface. However, some pores were not totally filled by PPy particles and it generated a small roughness, which is smaller than the other composites. Yet, a larger roughness for a same surface area increases the specific surface of a material, which is a characteristic sought for battery applications.

\subsection{Water Contact Angle and Sorption Kinetics}

The contact angles and the time dependence of the synthesized films were investigated by the dynamic contact angle measurements over time using static sessile drop method. The measured values a re shown in the Table 1 and in the Figure 3.

Table 1. Roughness, Contact angle and conductivity of each composite

\begin{tabular}{|l|ccc|}
\hline & $\begin{array}{c}\text { Roughness } \\
(\mathbf{R a})\end{array}$ & $\begin{array}{c}\text { Contact Angle } \\
\left({ }^{\circ}\right)\end{array}$ & $\begin{array}{c}\text { Conductivity } \\
(\mathbf{S} / \mathbf{c m})\end{array}$ \\
\hline TOCN/PPy-1 & $4.14 \pm 0.05$ & $73.6 \pm 1.1$ & $51.6 \pm 5.1$ \\
TOCN/PPy-2 & $4.07 \pm 0.28$ & $77.8 \pm 1.4$ & $47.6 \pm 1.9$ \\
TOCN/PPy-3 & $2.83 \pm 0.19$ & $79.6 \pm 0.4$ & $21.7 \pm 1.7$ \\
\hline
\end{tabular}




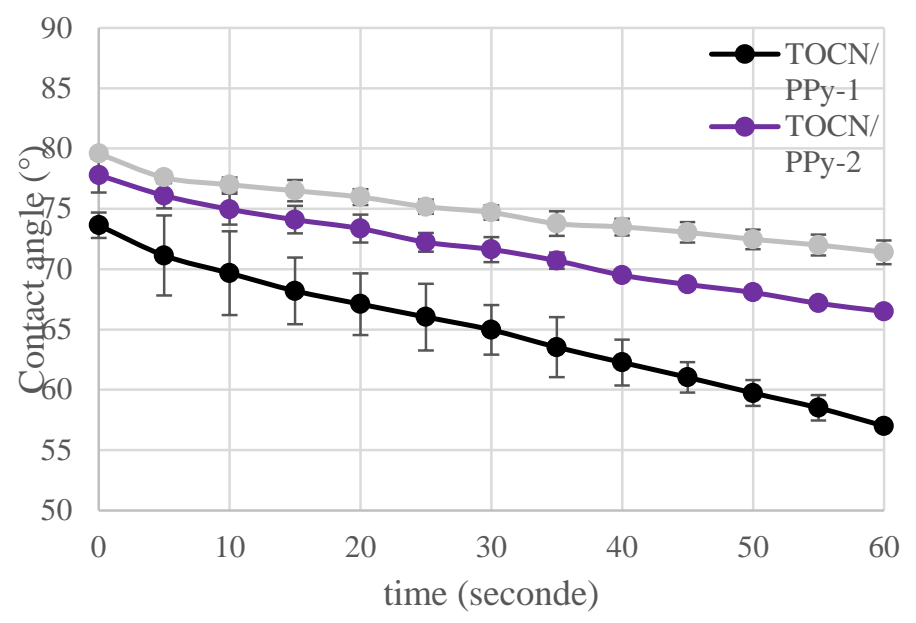

Figure 3. Variation of the contact angle as a function of time

It was found that the starting and decreases contact angles depend on the mat composition. TOCN material is known to have hydrophilic character with contact angle between $30-50^{\circ}[29,30]$. So the apparent contact angles of TOCN/PPy are larger than those with TOCN material. The incorporation of polypyrrole into the fibers resulted in hydrophobization of the composite (73.6-79.6 ${ }^{\circ}$, which is explained by the presence of the aliphatic chain of the particles of PPy. However, as seen from Figure 3, the method used has an important influence on the water contact angle and sorption kinetics. Indeed, TOCN/PPy-3 shows better values than two others. The presence on the surface of PPy layer increases the hydrophobic character and help to slow the composite wetting. After droplet deposition the contact angle decreased slower than TOCN/PPy-2 and TOCN/PPy-1. The first method led to more rapid absorption of the droplet by the composite (Figure 3) (twice as fast than the third method). This phenomenon can be explained by the composite roughness and the polymerization in solution which did not create a perfect PPy layer on the composite surface. So hydrophilic fibers of TOCN are more easily accessible to droplets and the resulting absorption is faster. The grafting of N-(3-aminopropyl)pyrrole gave the increase in hydrophobic character $\left(77.8^{\circ}\right)$, which is explained by the covalent bonds between TOCN fibers and PPy chains. For this reason, TOCN fibers are better protected by PPy chains which are closer than by electrostatic interaction (TOCN/PPy-1).

\subsection{Electrical Conductivity}

Composites electrical conductivities are presented in Table 1. Cellulose is known to be a high electrical insulator [11]. However, due to the PPy presence, the composites show a good electrical conductivity. This behaviour is attributed to the formation of a continuous conducting PPy layer which hides the cellulose insulating character. Coating homogeneity is very important to promote electrons transfer, thus enabling good conductivity. TOCN/PPy-1 and TOCN/PPy-2 lead to the best conductivity with a conductivity of around $50 \mathrm{~S} / \mathrm{cm}$ which is very good for a semi-conductor. Indeed, these results obtained are superior to those obtained with tunicate cellulose and PPy via the in situ oxidative chemical polymerization $(10 \mathrm{E}-3 \mathrm{~S} / \mathrm{cm})[30]$ or with bacterial cellulose $(2.7-3.9 \mathrm{~S} / \mathrm{cm})[31,32]$. Cellulose fibers which are coated by PPy particles by electrostatic or covalent bond confer a better conductivity than multilayer composite (TOCN/PPy-3). This difference in results can be explained by discontinuities between PPy particles in the layer surface, as seen in Figure 2c. In this case, the fibers are not coated individually and when there is a discontinuity, the cellulose insulating character affects the overall composite conductivity. So, the total homogeneity of PPy layer is very important to keep a good conductivity. 


\subsection{Thermal Properties}

The thermogravimetric curves are presented in Figure 4 and show clear differences between all composites. All composites contained a few trace of moisture which were eliminated at $110{ }^{\circ} \mathrm{C}$. Beyond weight percentage values, the general trends of the TOCN/PPy-1 and TOCN/PPy-2 curves are similar. We can see a first big weight loss of these composites between 200 and $320{ }^{\circ} \mathrm{C}$, where the corresponding weight loss was attributed to the destruction of the crystalline region and the decomposition of amorphous TOCN. The second weight loss corresponds to the degradation process of the PPy matrix. However, the grafting of PPy chains seems to confer a thermal advantage since we can observe a later decomposition of the composite TOCN/PPy-2 compared to TOCN/PPy-1. Moreover, it is possible to see that the TOCN/PPy-2 presents a slightly higher stability than the two other composites beyond 600 ${ }^{\circ} \mathrm{C}$. In comparison TOCN/PPy-3 shows a higher thermal stability with a first decomposition between $240{ }^{\circ} \mathrm{C}$ and $370{ }^{\circ} \mathrm{C}$. This increased protection can be attributed to the presence of polypyrrole layer on the surface which protects the fibers against thermal degradation. Finally, it is possible to observe a slow degradation of PPy material between 450 to $900{ }^{\circ} \mathrm{C}$ until the complete degradation of TOCN/PPy3 .

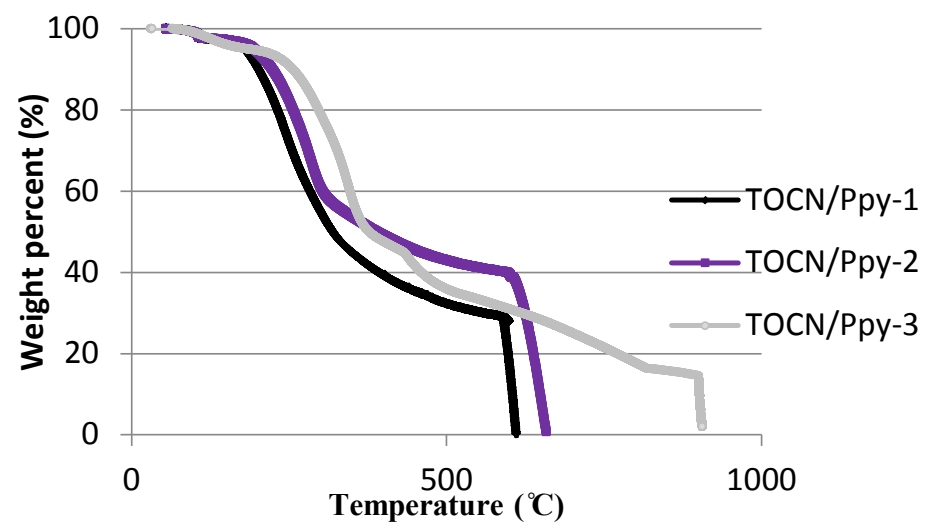

Figure 4. Thermogravimetric analysis of each composite

\subsection{Mechanical Properties}

The mechanical properties are shown in Table 2. Polypyrrole material alone is known to have poor mechanical properties. The tensile results are given after a clear breaking of the composite. The strength of the TOCN/PPy-3 can be attributed to strongest interactions, inter- and intra-molecular hydrogen bonding, occurring in the entangled structure of the cellulose fibers. In comparison, the presence of aliphatic chains around cellulose fibers, limits the mechanical properties of composites as it is possible to see with TOCN/PPy-1 and TOCN/PPy-2. This decrease (x2) can be attributed to the loss of interactions between fibers. Indeed, the presence of PPy particles interfering with the TOCN chains and reducing their interactions. However, the presence of grafted N-(3-aminopropyl)pyrrole, increases slightly the strength over TOCN/PPy-1. In a same way, TOCN/PPy-3 shows better young modulus than TOCN/PPy-2 and TOCN/PPy-1 due to higher crosslinking between TOCN fibers. TOCN film confers to TOCN/PPy-3, good flexible properties because it can be bent up to 180 degrees or even wound up. On the contrary, this structure presents a decreased elongation at break, which is expected with an increased Young modulus. Indeed, with less interaction between TOCN fibers, TOCN/PPy-1 increases the elongation at break (x1.5) over TOCN/PPy-3. The grafted N-(3-aminopropyl)pyrrole, TOCN/PPy-2, allows to obtain a composite with intermediate properties, achieving a good tensile strength while keeping an interesting elongation at break. 
Table 2. Mechanical properties (Young's Modulus, elongation at break, tensile stress) of each composite

\begin{tabular}{|l|ccc|}
\hline & $\begin{array}{c}\text { Young Modulus } \\
(\mathbf{G P a})\end{array}$ & $\begin{array}{c}\text { Tensile } \\
(\mathrm{MPa})\end{array}$ & $\begin{array}{c}\text { Elongation at } \\
\text { break (\%) }\end{array}$ \\
\hline TOCN/PPy-1 & $1.81 \pm 0.33$ & $28.7 \pm 2.1$ & $3.09 \pm 0.39$ \\
TOCN/PPy-2 & $2.52 \pm 0.28$ & $42.5 \pm 5.4$ & $2.55 \pm 0.14$ \\
TOCN/PPy-3 & $6.35 \pm 0.91$ & $65.6 \pm 0.5$ & $1.81 \pm 0.49$ \\
\hline
\end{tabular}

\section{Conclusion}

Flexible TOCN/PPy films were prepared by three different methods and their properties were evaluated. The chemical polymerization of pyrrole on surface of TOCN films shows the best mechanical and thermal properties. The results revealed a significant effect of TOCN film on the improvement of the mechanical properties of TOCN/PPy. The PPy layer allowed an increase in hydrophobic character of the composite. On contrary, TOCN/PPy-1 presented the worst mechanical properties due to the low number of interactions between cellulosic fibers. This method which consists to the polymerization in situ of pyrrole around the fibers, have demonstrated a better conductive capacity than TOCN/PPy-3. Thus, PPy particles distribution can be detrimental for the films mechanical properties. From our proposed methods, the grafting of $\mathrm{N}$-(3-aminopropyl)pyrrole was really interesting because it presented intermediate properties of all composites, and could be a good compromise between the mechanical and electrical properties. So we can conclude that the method used directly influences the composite conductivity and PPy particles distribution is detrimental for the films mechanical properties. Thus, for electrical applications such as in batteries, capacitors, sensors or solar cells for example, the use TOCN/PPy-1 and TOCN/PPy-2 seem to offer the best match but for flexible electrodes, TOCN/PPy-3 appears to be the best.

Acknowledgements. Lignocellulosic Materials Research Center (LMRC), Centre de recherche sur les matériaux renouvelables (CRMR), Fonds de recherche du Québec - Nature et technologies (FRQNT) and the Natural Science and Engineering Research Council of Canada (NSERC).

\section{Reference}

1. Ambade R. B., Ambade S. B., Shrestha N. K., Nah Y. C, Han S. H., Lee W. and Lee S. H., "Polythiophene infiltrated TiO2 nanotubes as high-performance supercapacitor electrodes", Chem. Commun., 2013, vol. 49, pp. $2308-2310$.

2. Wang, Z., Tammela, P., Zhang, P., Strømme, M.and Nyholm, I. "Nanocellulose coupled flexible polypyrrole@graphene oxide composite paper electrodes with high volumetric capacitance", J. Mater. Chem. A, 2014, vol. 2, pp. 7711-7716.

3. Molina, J., Fernández, J., Del Río A.I, Lapuente, R., Bonastre, J. and Cases, F., "Stability of Conducting Polyester/Polypyrrole Fabrics in Different pH Solutions, Chemical and Electrochemical Characterization", Polym. Degrad. Stab., 2010, vol. 95, pp. 2574-2583.

4. Chougulea, M. A., Pawar, S. G., Godse, P. R., Mulik, R. N., Sen, S. and Patil, V. B. "Synthesis and Characterization of Polypyrrole (PPy) Thin Films", Soft. Nanosci. Lett., 2011, vol. 1, pp. 6-10.

5. Shinde, S. and Gund, G. "Morphological Modulation of Polypyrrole Thin Films Through Oxidizing Agents and Their Concurrent Effect on Supercapacitor Performance", Electrochim. Acta., 2014, pp. 119, 1-10.

6. Sangawar, V.S. and Moharil, N.A. "Study of electrical, thermal and optical behavior of polypyrrole filled PVC: PMMA thin film thermoelectrets", Chem. Sci. Trans., 2012, vol. 1, pp. 447-455.

7. Ionescu, R. E., Abu-Rabeah, K., Cosnier, S., Durrieu C., Chovelon, J-M. and Marks, R. S. "Amperometric algal Chlorella vulgaris cell biosensors based on alginate and polypyrrole-alginate gels", Electroanalysis, 2006, vol. 18, pp. 1041-1046.

8. Zare, E. N., Lakouraja, M. M. and Mohsenib, M. "Biodegradable polypyrrole/dextrin conductive nanocomposite, Synthesis, characterization, antioxidant and antibacterial activity", Synth. Met., 2014, vol. 187, pp. 9-16. 
9. Sasso, C., Beneventi, D., Zeno, E., Petit-Conil, M., Chaussy, D. and Belgacem, M.N., "Carboxymethylcellulose, A conductivity enhancer and film-forming agent for processable polypyrrole from aqueous medium", Synth. Met., 2011, vol. 161, pp. 397-403.

10. Cabuk, M., Alan, Y., Yavuz, M. and Unal, H. I. Synthesis, "Characterization and Antimicrobial Activity of Biodegradable Conducting Polypyrrole-Graft-Chitosan Copolymer", Appl. Surf. Sci., 2014, vol. 318, pp. 168-175.

11. Jradi, K., Bideau, B., Chabot, B. and Daneault, C. "Characterization of Conductive Composite Films Based on TEMPO-Oxidized Cellulose Nanofibers and Polypyrrole", J. Mater. Sci., 2012, vol. 47, pp. 3752-3762.

12. Ding, C., Qian, X. and Yu, G., "A Dopant Effect and Characterization of Polypyrrole-Cellulose Composites Prepared by In Situ Polymerization Process", Cellulose., 2010, vol. 17, pp. 1067-1077.

13. Sasso, C., Zeno, E., Petit-Conil, M., Chaussy, D., Belgacem, M.N., Tapin-Lingua, S. and Beneventi, D. "Highly Conducting Polypyrrole/Cellulose Nanocomposite Films with Enhanced Mechanical Properties", Macromol. Mater. Eng., 2010, vol. 295, pp. 934-941.

14. Carlsson, D. O., Mihranyan, A., Strømme, M. and Nyholm, L. "Tailoring Porosities and Electrochemical Properties of Composites Composed of Microfibrillated Cellulose and Polypyrrole", RSC Adv., 2014, vol. 4, pp. 8489-8497.

15. Olsson, H., Nyström, G., Strømme, M., Sjödin, M. and Nyholm, L. "Cycling Stability and Self-Protective Properties of a Paper-Based Polypyrrole Energy Storage Device", Electrochem Commun., 2011, vol. 13, pp. 869871.

16. Shukla, S. K. "Synthesis and characterization of polypyrrole grafted cellulose for humidity sensing", Inter. J. Biolog. Macromol., 2013, vol. 62, pp. 531-536.

17. Mihranyan, A., Nyholm, L., Garcia-Bennett, A. E. and Strømme, M. "A Novel High Specific Surface Area Conducting Paper Material Composed of Polypyrrole and "Cladophora" Cellulose", J. Phys. Chem. B,. 2008, vol. 112, pp. 12249-12255.

18. Cai, J., Kimura, S., Wada, M. and Kuga, S. "Nanoporous cellulose as metalnanoparticles support", Biomacromol., 2008, vol. 10, no. 1, pp. 87-94.

19. Olsson, H., Carlsson, D. O., Nyström, G., Sjödin, M., Nyholm, L. and Strømme, M. "Influence of the Cellulose Substrate on the Electrochemical Properties of Paper-based Polypyrrole Electrode Materials", J. Mater. Sci., 2012, vol. 47, pp. 5317-5325.

20. Carlsson, D. O., Sjödin, M., Nyholm, L. and Strømme, M. "A Comparative Study of the Effects of Rinsing and Aging of Polypyrrole/Nanocellulose Composites on Their Electrochemical Properties", J. Phys. Chem. B., 2013, vol. 117, pp. 3900-10.

21. Paquin, M., Loranger, E., Hannaux, V., Chabot, B. and Daneault, C. "The Use of Weissler Method for Scale-Up a Kraft Pulp Oxidation by TEMPO-Mediated System from a Batch Mode to a Continuous Flow-Through Sonoreactor", Ultrason Sonochem., 2013, vol. 20, pp. 103-8.

22. Rattaz, A., Mishra, S., Chabot, B. and Daneault, C. "Cellulose Nanofibres by Sonocatalysed-TEMPOOxidation", Cellulose, 2011, vol. 18, pp. 585-593.

23. Syverud, K. and Stenius, P. "Strength and barrier properties of MFC films", Cellulose, 2009, vol. 16, pp. 75-85.

24. Saito, T. and Isogai, A. "TEMPO-Mediated Oxidation of Native Cellulose. The Effect of Oxidation Conditions on Chemical and Crystal Structures of the Water-Insoluble Fractions", Biomacromol., 2004, 5, 1983-1989.

25. Lee, J. Y. and Schmidt, C. E. "Pyrrole-hyaluronic acid conjugates for decreasing cell binding to metals and conducting polymers", Acta Biomat., 2010, vol. 6, pp. 4396-4404.

26. Hu, W., Chen, S., Yang, Z., Liu, L. and Wang, H. "Flexible electrically conductive nanocomposite membrane based on bacterial cellulose and polyaniline", J. Phys. Chem. B, 2011, 115, pp. 8453-8457.

27. Naji, A., Cretin, M., Persin, M. and Sarrazin, S. "Electrical characterization of the ionic interactions in N-[3(dimethylpyridyl-2-yl) aminopropyl] polypyrrole and N-(3-aminopropyl) polypyrrole membranes", J. Membrane Sci., 2003, 212, pp. 1-11.

28. Bideau, B., Cherpozat, L., Daneault, C. and Loranger, E. "A conductive nanocomposite based on TEMPO oxidized cellulose and poly(N-3-aminopropylpyrrole-co-pyrrole)", Ind. Crop. Prod, DOI:10.1016/j.indcrop2016.06.003.

29. Bideau, B., Bras, J., Saini, S., Daneault, C. and Loranger, E. "Mechanical and antibacterial properties of a nanocellulose composite multilayer”, Mat. Sci. Eng. C Mater. Biol. Appl., 2016, vol. 69, pp. 977-984. 
30. Zhang, D., Zhang, Q., Gao, X. and Piao, G. "A Nanocellulose Polypyrrole Composite Based on Tunicate Cellulose", Int. J. Polym. Sci., 2013, vol. 2013, pp. 1-6.

31. Xu, J., Zhu, L., Bai, Z., Liang, G., Liu, L., Fang, D. and Xu, W. "Cotton fabrics coated with lignosulfonatedoped polypyrrole for flexible supercapacitor electrodes", Org. Electron., 2013, vol. 14, pp. 3331-3338.

32. Muller, D., Rambo, C. R., Porto, L. M., Schreiner, W. H. and Barra, G. M. O. "Structure and properties of polypyrrole/bacterial cellulose nanocomposites", Carbohyd Polym., 2013, vol. 94, pp. 655-662. 